\title{
A ABORDAGEM FONÉTICA DE DADOS CLÍNICOS
}

\section{CAMARGO, Zuleica*}

Pontifícia Universidade Católica de São Paulo - Laboratório Integrado de Análise Acústica e Cognição (LIAAC)

\begin{abstract}
This study aims at presenting a review, from the methodological point of view, of procedures and demands related to the phonetic approach of clinical data. The possibility of taking into account the interface between speech perception and production may be productive when analyzing data collected from clinical settings or developing researches in which the central questions arose from the clinical practice. The dynamics aspects of speech are considered. The series of studies reported reinforces the indication of a highly integrated and cooperative approach among the several research areas concerned on speech production and perception. The capability of producing speech under disorders caused by damages in phonatory and auditory systems can stimulate researchers to develop approaches that could influence theoretical models, rehabilitation tools and speech technologies development.
\end{abstract}

Keywords: speech therapy; speech acoustics; speech perception; voice quality. 


\section{Introdução}

A abordagem fonético-acústica de dados advindos da clínica fonoaudiológica tem se configurado como uma possibilidade de leitura atenta e detalhada dos mecanismos subjacentes à geração do sinal de fala ${ }^{1}$ registrado, muitas vezes fruto de mecanismos compensatórios desenvolvidos na tentativa de superar alterações de natureza estrutural e/ou funcional do aparelho fonador e do sistema auditivo.

A leitura de dados clínicos revela a possibilidade de uma relação profícua entre Fonoaudiologia e Ciências Fonéticas, em que, num extremo, temos a possibilidade de detalhamento de mecanismos que podem ser determinantes da manifestação clínica, permitindo um planejamento terapêutico diferenciado. No outro extremo, contamos com a condição de aprofundar a leitura de dados de variabilidade de fala. Situada entre os extremos citados, encontramos uma vasta gama de manifestações que interessam tanto àqueles que se dedicam a atividades clínicas, quanto àqueles envolvidos com o estudo da variabilidade da fala e com a possibilidade de adoção de modelos de natureza dinâmica (Gregio et al, 2006).

Neste concepção, ambos os campos de conhecimento beneficiam-se da abordagem da plasticidade do aparelho fonador em se adaptar a demandas advindas de uma vasta gama de alterações, além das possibilidades expressivas da fala, outra abordagem refinada permeada pela Fonética Acústica e enfocada no universo de atuação fonoaudiológica.

A consideração das manifestações clínicas sob a perspectiva fonética conclama os fonoaudiólogos a romperem a clássica dicotomia estabelecida entre normalidade x alteração, a qual, além de não contribuir para a compreensão mais refinada das alterações de fala, e de suas possibilidades de abordagem clínico-terapêutica, leva a uma simplicação da natureza dinâmica dos processos de percepção e de produção da fala.

Neste contexto, o nosso enfoque a respeito da possibilidade de "cura" das alterações deve superar a consideração de um "ideal de normalidade”, no sentido de que comparar o comportamento de um falante a tal ideal pode impor limitações na consideração de suas reais possibilidades: as condições anátomo-fisiológicas presentes, aquelas de sua adaptação à situação da alteração de fala, de seu conhecimento da língua e das possibilidades e restrições inerentes (Camargo, 2004).

Tal concepção permite superar outras dicotomias, tais como fala x voz e fonética x fonologia. Entender a manifestação em si e orientar o falante quanto às possibilidades e às limitações presentes parece ser a solução mais indicada (Marchesan, 2004, 2004a). Além disso, a adoção de modelos de

\footnotetext{
${ }^{1}$ Adotamos o termo sinal de fala como o resultado dos complexos processos nos planos linguístico, fisiológico, acústico e perceptivo, em que são congregados os elementos segmentares e prosódicos. Sendo a qualidade vocal um elemento prosódico, os aspectos clínicos da voz também estão comtemplados no termo sinal da fala. Para corresponder às questões de abordagem clínica nas especialidades na atuação fonoaudiológica, mencionaremos fala no sentido de conjugar fala e voz do ponto de vista clínico, o que equivaleria ao sinal de fala-voz. Os aspectos tradicionalmente enfocados como "articulatórios" na clínica fonoaudiológica (nas especialidades de motricidade orofacial e de linguagem) corresponderiam aos elementos segementares, enquanto aqueles da atuação em especialidades de voz e na linguagem, e, particularmente, na área de fluência, corresponderiam ao enfoque dos elementos prosódicos. Não se pretende estabelecer uma divisão enetre estes elementos, mas valorizar sua possibilidade de interação, conforme indicado em Albano et al. (1997). Adotamos uma perspectiva de associação de informações de natureza perceptiva, acústica e fisiológica, na busca pela descrição integrada do sinal de fala (Stevens, 1989, 1998; Johnson, 2003).
} 
natureza dinâmica permite um olhar diferenciado sobre estas manifestações, enfocadas mais propriamente como gradientes de percepção e de produção de fala (Madureira et al., 2002; BarzaghiFicker, 2003; Camargo et al., 2003; Mendes, 2003; Vieira et al., 2004; Berti, 2006; Gregio, 2006; Bonatto, 2007; Pereira, 2007; Barzaghi-Ficker et al., 2008; Soares, 2009; Lima-Gregio, Barbosa, 2010; Oliveira, 2011; Camargo et al., 2011, Marchesan et al., 2011).

Diante da possibilidade de adoção de referencial teórico que prima pelas relações entre a percepção e a produção de fala, nossas indagações direcionam-se às demandas metodológicas advindas da possibilidade de desenvolvimento de pesquisas a partir de dados clínicos: como planejar a coleta de dados? como conceber o corpus de pesquisa? Há necessidade de grupo controle ou da adoção de estudos que tenham trabalhado com as mesmas variáveis na população sem alterações? Como enfocar as correlações entre as esferas perceptiva, acústica e fisiológica?

No cenário nacional, destacamos modelos de natureza dinâmica, como o esboço da fonologia acústico-articulatória para o PB (Albano, 2001) e do modelo dinâmico de ritmo (Barbosa, 2006).

Neste ponto, surge a questão central de nossa exploração neste artigo: de que forma os preceitos fonético-acústicos permitem-nos analisar dados de fala de forma a não nos atermos à abordagem dicotomizante entre normalidade e alteração? Tais desafios nos são postos a cada tentativa de enfoque de dados de alteração de fala (em suas vertentes da percepção e da produção), uma vez que uma primeira possibilidade reside na adoção de dados de um grupo controle, ou de um indivíduo referência, para se estimar o nível de alteração.

Diante desse universo de possibilidades e de limitações, em que a exploração do sinal acústico da fala emerge como uma alternativa promissora de estudos e, ao mesmo tempo, como objeto complexo em termos de registro, processamento e análise, este artigo visa discorrer sobre aspectos metodológicos de estudos que possam trazer uma contribuição ao enfoque dos processos de diagnóstico e de planejamento terapêutico. Para tanto, a alteração de fala deixa de ser considerada como um desvio numérico em relação a uma situação supostamente irreal: a fala ideal. Passa a ser enfocada enquanto produção sonora decorrente de alterações orgânicas e/ou funcionais em toda a extensão do aparelho fonador e/ou do sistema auditivo.

\section{Uma reflexão à luz da metodologia dos estudos fonético-acústicos de dados clínicos}

Nossa incursão aos referenciais fonéticos iniciou-se pela busca de compreensão da implementação de ajustes desenvolvidos por falantes com alterações de maior porte no aparelho fonador, provocadas por tratamentos oncológicos (cirurgias, combinações de radioterapia e quimioterapia) em cabeça e pescoço. Numa exploração inicial de grupo de falantes submetidos a laringectomias parciais verticais ${ }^{2}$,

\footnotetext{
${ }^{2}$ Laringectomias parciais verticais referem-se a cirurgias da laringe em que parte da sua estrutura é removida, de maneira a permitir o desempenho das funções de respiração, proteção para deglutição e fonação, porém com comprometimentos do conjunto de funções em graus variados. Consistem na remoção parcial ou total de uma prega vocal e, por vezes, de parte da prega vocal contralateral. Nestes casos, a remoção cirúrgica ocorre de forma mais intensa de um lado da laringe (direito ou esquerdo).
} 
pudemos nos beneficiar da adoção de abordagem integrada de dados da percepção, fisiologia e acústica, como forma de diferenciar os padrões funcionais compensatórios desenvolvidos por falantes submetidos a tratamentos similares, porém com resultados funcionais variados (Camargo, 1996).

Tal exploração inicial nos motivou a prosseguir pela análise de alterações do aparelho foandor, como no caso das incompetências glóticas, tendo como base descrições da percepção (qualidade vocal e reconhecimento de fala), suas correspondências acústicas (de curto e de longo termo) e fisiológicas (eletroglotografia, videolaringoestroboscopia e videoquimografia) (Camargo, 2002).

Enfocamos um grupo de indivíduos portadores de alterações de mecanismos glóticos justamente pela possibilidade de estabelecimento de grande variedade de compensações, as quais não se restringem à porção glótica do aparelho fonador, ou seja, ao nível que concentra a principal limitação à produção sonora (Camargo, 2002). Portanto, o estudo centrou-se nos ajustes individuais implementados em seu aparelho fonador durante a produção da fala, respaldado pela teoria acústica da produção da fala (Flanagan, 1955, 1957; Fant, 1970) e pelo modelo fonético de descrição da qualidade vocal (Laver, 1980), com incorporação do roteiro Vocal Profile Analysis Scheme - VPAS, em sua verão resumida (Laver, 2000).

Os desafios metodológicos impostos residiram na seleção do grupo estudado, na concepção de corpus e na eleição de procedimentos para integração dos dados das esferas perceptiva, acústica e fisiológica, uma vez que a coleta de dados envolvia informações de naturezas distintas.

Em termos do grupo estudado, não intentamos desenvolver uma pesquisa quantitativa baseada em análise de limitados parâmetros e amplo número de falantes. Em contraposição, optamos por um grupo de quatro indivíduos em atendimento clínico por disfonia de fator desencadeante comum (incompetência glótica, por paralisia de prega vocal ou fibrose laríngea) e um indivíduo referência da mesma faixa etária e sexo, sem almejarmos propor generalizações sobre a produção sonora na situação de disfonia, mas, em contraposição, ilustrar as várias facetas da alteração da qualidade vocal.

Vale ressaltar que a referência à fonação na ausência de alterações do aparelho fonador passou a ser o ponto de apoio necessário para que se considerasse, dentre os ajustes, aqueles que são impostos pela mudança de atividade em decorrência de incompetência glótica, subsequente à instalação de paralisia unilateral ou de fibrose de prega vocal. Integrou um ponto no contínuo sonoro (Mathieson, 2000) que se estende desde a fonação com sonoridade e sem ruídos, até aquela situação em que a fonte de sonoridade é completamente substituída por ruído turbulento e a sonoridade praticamente ausente.

Como justificativa para tal procedimento, destacamos que a adoção de indivíduo referência para estudos de base fonético-acústica também envolveu o levantamento de particularidades da emissão oral de falantes de determinada comunidade linguística e, nesta situação delineada, de ausência de condições características de quadro de distúrbios de fala, à semelhança de outros estudos (Camargo, 1996, 2002; Barzaghi-Ficker, 2003; Mendes, 2003; Pereira, 2007).

Em termos do corpus, num momento em que maioria dos estudos ainda enfocava, quase que exclusivamente, a emissão de vogais sustentadas, a análise de longo termo poderia ser comprometida 
pela inexistência de material padronizado para tal finalidade em nossa língua. Optamos por compor texto que resumisse várias das necessidades de nosso grupo de estudos, como uma primeira etapa de concepção de instrumentos a serem utilizados em pesquisas subsequentes no campo da Fonética Perceptiva e Acústica.

Partimos para elaboração de estímulo para leitura: texto com presença de sons orais, nasais, vozeados e não vozeados (total de 209 palavras), com duração mínima prevista de 90 segundos (quando em leitura em taxa de elocução elevada). $\mathrm{O}$ avanço das tecnologias de análise acústica ainda não nos permitia, com facilidade, a seleção apenas do segmentos vozeados, como hoje se faz, de maneira que elaboramos um segundo texto, com predomínio de sons vozeados (145 palavras). As medidas acústicas de longo termo de três repetições de leituras de ambos os textos foram comparadas em um dos falantes (disfônicos) do grupo estudado, por meio da análise de componentes principais e construção de clusters, de forma a revelar similaridade de achados para as leituras dos dois textos elaborados (Camargo, 2002).

A preocupação nesta etapa recaía sobre a possibilidade de segmentos não vozeados presentes no texto sinalizarem, erroneamente, eventos de ajustes de escape de ar e soprosidade do ponto de vista laríngeo. Superada esta etapa, prosseguimos com análise dos dados do texto misto de 209 palavras para todos os falantes do grupo estudado. Tal instrumento também foi empregado em estudos subsequentes (Lima, 2008; Camargo, Madureira, 2009; Giannini, 2010).

Como fatores limitantes, destacamos que tal proposta de corpus dificultou-nos a abordagem de alguns índices acústicos, como extração de frequências formânticas de vogais e de medidas de voiceonset-time (VOT) das consoantes plosivas, uma vez que o contexto da inserção dos segmentos vocálicos e consonantais não era controlado, como no caso do emprego de sentenças-veículo, como em Mendes (2003), Barzaghi-Ficker et al. (2008) para vogais e por Barzaghi-Ficker (2003), Bonatto (2007) e Pereira (2007) para consoantes.

Do ponto de vista da análise de dados, o desafio residiu na tentativa de corresponder as esferas relevantes da estrutura acústica do sinal aos atributos perceptivos e fisiológicos. Contamos com apoio de softwares para análise eletroglotográfica (Vieira et al., 2002), propiciando maior acurácia na leitura de dados numa situação geralmente contraindicada, a paralisia ou fixação de prega vocal.

As limitações residem no fato de que a qualidade vocal engloba várias dimensões, de forma que os achados de correlação entre um determinado parâmetro acústico e um respectivo julgamento perceptivo podem não sinalizar uma relação estreita. Flanagan (1957), um dos precursores de estudos da acústica da fala e de suas relações com a percepção, apontava a dificuldade de se fazer corresponder uma medida acústica à qualidade auditiva, diante do desconhecimento de outros fatores que poderiam colaborar para o efeito sonoro detectado.

Reforçando tais aspectos da complexidade das correlações entre percepção e acústica da qualidade vocal, Kreiman, Gerrat (2000) apresentaram uma revisão crítica dos estudos, especialmente daqueles centrados nos aspectos glóticos da emissão, salientando que a qualidade vocal resultaria da 
interação entre o sinal acústico e um ouvinte, de maneira que a "qualidade" seria evocada no sistema perceptivo do ouvinte e não puramente na estrutura do sinal. A partir desta argumentação discorreram sobre muitas das limitações na leitura de dados de correspondências perceptivas e acústicas e reforçaram o cuidado na seleção da abordagem estatística dos dados. Neste ponto, chegaram a revisar dados de estudos publicados com variação da metodologia estatística empregada e apontando métodos de análise multivariada como os mais promissores.

Retomando os dados do estudo de Camargo (2002), adotamos procedimento de análise de componentes principais e de construção de clusters para a análise acústica de longo termo. Para avançar no enfoque da disfonia, enquanto manifestação de caráter adaptativo, defendemos a posição de que o quadro a que ela se refere implica diversos graus de mobilização para a tentativa de manutenção do produto sonoro, mobilização essa que revela a plasticidade do aparelho fonador em face de alterações em sua estrutura. Nas etapas de análise, as emissões do grupo de disfônicos e indivíduo referência foram analisados em conjunto e separadamente (apenas disfônicos), como forma de se estimar as gradiências na percepção e na produção (Mathieson, 2000).

Diante desta abordagem, o grupo estudado revelou a fascinante condição de adaptação do aparelho fonador diante de limitações de ordem de mobilização e vibração dos tecidos da região laríngea e as variadas resultantes sonoras possíveis, a partir de gestos particularizados e integrados para cada falante. Tais gestos podem ser abordados como forma de cumprir a necessidade crucial de sonorização da coluna aérea e transmissão da energia sonora, como um importante requisito da comunicação oral.

Podemos considerar que, diante de manifestações de base comum, enquanto quadro clínico, desvendamos ações diferenciadas com maior ou menor efetividade em termos da adaptação da função vocal, condição visada pela intervenção terapêutica. Tal consideração nos foi possível a partir da concepção fonética adotada,

Tais achados nos conduziram à reflexão de que a exploração da qualidade vocal enquanto um traço recorrente da característica da emissão do falante deveria ser cuidadosamente dimensionada, no sentido de se traduzir em abordagens mais fidedignas de avaliação, a revelarem os fatores subjacentes que caracterizam o sofrimento dos indivíduos disfônicos. Tal aspecto inclui a demanda por estabelecimento de consistente treinamento auditivo na formação do fonoaudiólogo e das possibilidades de atuação em avaliação e assistência terapêutica, com importantes contribuições aos campos experimental e clínico e, inclusive para adaptação da formação do profissional fonoaudiólogo.

Na continuidade de exploração do tema e trabalhando com os dados gerados (Camargo et al., 2003; Camargo, Madureira, 2009), iniciamos nosso empenho na implementação e adaptação de instrumento de avaliação perceptiva da qualidade vocal com motivação fonética para o português brasileiro, justamente pelos desafios metodológicos anteriormente relatados. 
Iniciamos projeto de abordagem fonética da qualidade vocal, de forma a apresentar a versão VPAS- PB $^{3}$ (Camargo, Madureira, 2008), juntamente a proposta de corpus para coletas de amostras de qualidade vocal, composto de emissões semi-espontâneas (de caráter dirigido, como, por exemplo, o relato sobre a cidade onde o falante nasceu), bem como leitura de sentenças-chave, baseadas no conceito de segmentos-chave, enquanto segmentos vocálicos e consonantais susceptíveis aos efeitos de ajustes de qualidade vocal, conforme bases de modelo fonético de Laver (1980), e diretrizes para uso do VPAS em sua versão original (Mackenzie-Beck, 2005; Laver, Mackenzie-Beck, 2007).

A partir do trabalho com o referido instrumento, além das novas demandas advindas desta etapa de trabalhos, passamos a trabalhar no sentido de estruturação de banco de dados de qualidades vocais (Camargo, Madureira, 2008), de forma a gradativamente explorar as possibilidades de correlações de dados perceptivos e acústicos (Blaj et al., 2008; Rusilo et al., 2011; Camargo, Madureira, 2012), inclusive quanto à revisão crítica do corpus desenvolvido (Camargo et al., 2011) e a particularidades entre gêneros (Camargo et al., 2012).

O trabalho constante na ampliação do banco de dados, na formação de juízes habilitados ao uso do VPAS-PB e na exploração de dados de natureza perceptiva e acústica conduziram-nos à busca por aprimoramento de análise das correlações, bem como à definição de um conjunto de parâmetros acústicos que pudesse refletir os aspectos recorrentes da qualidade vocal. A demanda pela bateria de variáveis acústicas nos foi possibilitada pelo uso do script ExpressionEvaluatorSG (Barbosa, 2009), que permite a extração de um conjunto de medidas absolutas e normalizadas de frequência fundamental (f0, primeira derivada de f0, intensidade, declínio espectral e espectro de longo termo).

Diante da incorporação deste instrumento, que permite a análise de trechos de fala e não apenas de segmentos isolados, por meio da etiquetagem de enunciados, pudemos trabalhar com uma nova perspectiva de descrição de correlatos acústicos (Pessoa et al., 2009, 2011, 2012; Fernandes, 2011; Lima-Silva, 2012), a qual impulsionou o desenvolvimento e adaptação de procedimentos de análise estatística de natureza multivariada para o banco de dados em questão (Camargo, Madureira, 2010; Rusilo et al., 2011; Camargo et al., 2012a; Lima-Silva, 2012; Pessoa et al., 2012).

Certamente, ao lidarmos com dados de falantes com e sem alterações de fala, buscamos alternativas de análise que contemplem as demandas da população e dos estudos em questão, especialmente com destaque àqueles que não nos permitem retroceder a uma abordagem dicotomizante.

Em alguns de nossos estudos mais recentes, diante da metodologia descrita, pudemos estimar comportamentos dos indivíduos com alterações de fala, na tentativa de entender a particularidade daquele grupo, até que a demanda de caracterização de parâmetros acústicos seja efetivada para o nosso grupo de falantes do PB. Dessa forma, estes estudos não se pautaram em comparações com

\footnotetext{
${ }^{3}$ Vocal Profile Analysis Scheme (VPAS) refere-se a um instrumento de avaliação perceptiva de qualidade vocal (Laver et al., 1981), desenvolvido a partir de modelo fonético de descrição da qualidade vocal (Laver, 1980), tendo sido adaptado para o português brasileiro: VPAS-PB (Camargo, Madureira, 2008). O material instrutivo desenvolvido com a finalidade de incorporação do VPAS-PB inclui a proposta de corpus para coleta de dados.
} 
população sem alteração de fala, mas detalharam os comportamentos dos falantes, com base em descrições perceptivas (Fernandes, 2011) e integradas à acústica (Pessoa et al., 2009, 2011, 2012; Camargo et al., 2012a; Lima-Silva, 2012).

Com o desenvolvimento dos estudos em expressividade da fala, esboça-se a tentativa de obtenção de dados de fala "natural" ou espontânea, muitas vezes limitada em termos de coletas por questões éticas, em complementação às coletas que ocorrem em situações experimentais controladas (Xu, 2010).

Neste campo, outra possibilidade de enfoque, e de composição de corpus por parte de fonoaudiólogos reside em registros de trechos de sessões de terapia. Neste caso, particularmente, temos trabalhado com estruturação de banco de dados em coletas seriadas de amostras de fala em situações de terapia, com crianças usárias de implante coclear. Tal banco de dados tem permitido a incorporação de amostras de fala infantil de natureza semiespontânea (respostas a estratégias terapêuticas) e gerado estudos que apresentam dados do(s) mesmo(s) falante(s) em recortes longitudinais (Pessoa et al., 2009, 2012). Abrimos mão da padronização na coleta de amostras para assumirmos a possibilidade de registrar produções espontâneas de crianças com implante coclear. Tal metodologia tenta contemplar a demanda acima apontada.

Em outras explorações, além da possibilidade de descrição com base na incorporação de um indivíduo referência, ou seja, sem o quadro clínico, da mesma faixa etária e sexo (Camargo, 2002; Barzaghi-Ficker, 2003; Mendes, 2003; Pereira, 2007), pode-se optar por um grupo controle (CukierBlaj, 2006; Oliveira, 2011), situação em que, ao mesmo tempo apresenta a abordagem das alterações à luz do referencial das Ciências Fonéticas, brinda-nos com a possibilidade de descrição das variáveis enfocadas para a população sem alterações de mesma faixa etária e sexo.

Diante desta lacuna, alguns fonoaudiólogos optaram por conduzir estudos de caracterização de parâmetros fonético-acústicos na população sem alterações de fala, como é caso de Bonatto (2007) para crianças e Gregio et al. (2011) para adultos, com o propósito de, futuramente, trabalharem com populações com alterações.

Neste constante empenho por caracterização das alterações sem meras comparações com a a normalidade, encontramos no modelo fonético um respaldo para enfoque de ajustes de qualidade vocal que foi recentemente adotado pelo grupo, na medida em que o banco de dados começa a ter um conjunto de amostras suficientes para cobrir a descrição de várias manifestações de ajustes possíveis ao aparelho fonador.

Nesta situação recente, contamos com a possibilidade de comparação de variáveis acústicas e perceptivas que nos garantam uma calibração entre várias estados de alteração, a partir da comparação de varíaveis relativas a ajustes neutros e não neutros de qualidade vocal, sem ter que, necessariamente, selecionar previamente a população por dividí-las em grupos. Esta concepção metodológica também permitirá estudar, com maior detalhamento, a questão dos graus de manifestação dos ajustes não neutros de qualidade vocal, acentuando-se que, ajustes não neutro não significa fala alterada, mas uma 
postura diferenciada daquela adotada como referência no modelo teórico: o ajuste neutro (Camargo et al., 2011).

O enfoque da percepção em nosso grupo não se ateve aos aspectos de qualidade vocal, aqui detalhados por ser a linha de pesquisa da autora. Em termos da percepção da fala alterada, outra possibilidade de abordagem que merece destaque refere-se à avaliação da fala por indivíduos com e sem alteração. Neste particular, o falante com alteração da fala também passa a exercer o papel de juiz de suas produções e daquelas do(s) falante(s) sem alaterações (Barzaghi-Ficker, 2003).

Diante das possibilidades esboçadas, cabe destacar que a caracterização fonético-acústicoarticulatória de variantes do português brasileiro (PB), tanto do ponto de vista segmental (Silva, Albano, 1999; Madureira et al., 2004; Gregio, 2006; Bonatto, 2007), quanto prosódico (Madureira, 1994; Barbosa, Madureira, 1999; Madureira, Barbosa, 2000; Madureira, 2002; Madureira, Diniz, 2002; Madureira, Fontes, 2003; Barbosa et al., 2005; Barbosa, 2006; Barbosa et al., 2007, 2008; Barbosa, Silva, 2012), em populações de adultos e crianças, é ainda incipiente.

No campo prosódico, a produção de nosso grupo destaca o empenho na adaptação de roteiro VPAS-PB (Camargo, Madureira, 2008). Tal produção resultou em possibilidades de abordagens de populações com alterações de fala, como é caso de deficientes auditivos e crianças usuárias de implante coclear (Pessoa et al., 2009, 2011, 2012) e de professores com disfonia (Fernandes, 2011; Lima-Silva, 2012), bem como de explorações no campo da expressividade de fala (Madureira, Camargo, 2010, Madureira, 2011).

As reflexões acima delineadas encontram respaldo na afirmação de Whalen (1999) de que: "toda teoria de fala deve explicar como os ouvintes podem relacionar uma articulação com um sinal acústico. O link entre percepção e produção é forte, imediato e inevitável na fala humana”.

Destacamos a necessidade de uma abordagem altamente integrada e cooperativa entre a diversas áreas de conhecimento voltadas ao enfoque da percepção e da produção de fala humana, com o intuito de contribuir para a formação de conhecimento sobre os complexos níveis de atividade que permeiam a interface da percepção e da produção sonora.

\section{Considerações Finais}

A capacidade extraordinária de produção de fala na vigência de alterações que podem, inclusive, resultar de verdadeiras "mutilações" do aparelho fonador e do sistema auditivo, tem servido de base para a reflexão dos pesquisadores sobre esta faculdade humana tão complexa. A leitura fonética de dados clínicos também pode auxiliar a concepção e o aprimoramento de modelos teóricos, uma vez que nos indicam situações extremas de uso do aparelho fonador, raramente presentes na fala cotidiana.

Tal nível de colaboração também enriquece a produção científica e a atuação clínica do fonoaudiólogo, que, ao incorporar a visão integrativa do sinal de fala, passa a compreender a complexidade das relações entre a percepção e a produção da fala e a refinar suas possibilidades de atuação com falantes com alterações de fala de natureza tão peculiares. 


\section{Agradecimentos}

Ao CEP-PUCSP (09/639). À Aline Pessoa, pela revisão do manuscrito.

\section{Referências}

Albano EC. O gesto e suas bordas: esboço de fonologia acústico-articulatória do português brasileiro. Campinas: Mercado de Letras; 2001.

Barbosa PA, Silva W. A new methodology for comparing speech rhythm structure between utterances: beyond typological approaches. In: Caseli H, Villavicencio A, Teixeira A, Perdigao F, editors. Computational processing of the Portuguese language. Berlim: Springer; 2012. v. 7243, p. 329-37.

Barbosa P. Incursões em torno do ritmo da fala. Campinas: Pontes, São Paulo: FAPESP; 2006.

Barbosa P, Coimbra RL, Madureira S, Moutinho LC. Análise contrastiva dos contornos prosódicos de duas variedades do português. In: Niemeyer $\mathrm{M}$, Hambye $\mathrm{P}$, editors. Proceedings of the XV Congrès International de Linguistique et de Philologie Romanes; 2008, Set 3-8; Innsbruck. Tubingen: D. Trotter; 2008. vol. 2, p. 45-52.

Barbosa PA, Lucente L, Silveira LS, Madureira S. Intonational patterning in the Paulista Brazilian Portuguese variety: two case studies in the framework of AMPER. In: II Congresso de Fonética Experimental; 2005. p. 117-30.

Barbosa P, Lucente L, Silveira L, Madureira S. Intonational patterning in the Paulista Brazilian Portuguese variety: two case studies in the framework of AMPER. In: Actas do III Congresso de Fonética Experimental; 2007; Santiago de Compostela e Xunta de Galicia: Universidade de Santiago de Compostela e Real Academia Galega; 2007. p. 117-31.

Barbosa P, Madureira S. Toward a hierarchical model of rhythm production: evidence from phrase stress domains in Brazilian Portuguese. In: Ohala JJ, Hasegawa Y, Ohala M, Granville D, Bailey AC, editors. Proceedings of the 14th International Congress of Phonetic Sciences; 1999 Ago 1-7; São Francisco. Berkeley: University of California; 1999. v. 1. p. 297-301.

Barbosa PA. Detecting changes in speech expressiveness in participants of a radio program In: Uther M, Moore R, Cox S, editors. Proceedings of Interspeech; 2009 Set 6-10; Brighton. Causal Productions Pty Ltd; 2009. v. 1, p. 2155-8.

Barzaghi-Ficker L. Produção e percepção das plosivas do português brasileiro: estudo fonéticoacústico da fala de um sujeito com deficiência auditiva. [Tese de doutorado]. São Paulo: Pontifícia Universidade Católica de São Paulo; 2003.

Barzaghi-Ficker L, Mendes BCA, Madureira S. Stressed and unstressed vowel production in hearingimpaired speech In: Barbosa P, Reis C, Madureira S, editors. Fourth Conference on Speech Prosody; 2008 Mai 6-9; Campinas. Campinas: Capes, Fapesp, CNPq; 2008. v. 1. p. 199-202. 
Berti LC. Aquisição incompleta do contraste entre /s/ e /S/ em crianças falantes do português brasileiro. [Tese de doutorado]. Campinas: Universidade Estadual de Campinas; 2006.

Bonatto MTRL. Vozes infantis: a caracterização do contraste do vozeamento das consoantes plosivas do português brasileiro na fala de crianças de 3 a 12 anos. [Tese de doutorado]. São Paulo: Pontifícia Universidade Católica de São Paulo; 2007.

Camargo Z. Parâmetros vocais e configurações laríngeas na fonação de indivíduos submetidos às laringectomias parciais verticais. [Dissertação de mestrado]. São Paulo: Pontifícia Universidade Católica de São Paulo; 1996.

Camargo Z, Madureira S. Voice quality analysis from a phonetic perspective: Voice Profile Analysis Scheme Profile for Brazilian Portuguese (BP-VPAS) In: Barbosa P, Reis C, Madureira S, editors. Fourth Conference on Speech Prosody; 2008 Mai 6-9; Campinas. Campinas: Capes, Fapesp, CNPq; 2008. v. 1. p. 57-60.

Camargo Z, Marchesan IQ, Oliveira LR, Svicero MAF, Pereira LCK, Madureira S. Lingual frenectomy and the alveolar tap production: an acoustic and perceptual study. Logopedics, Phoniatrics and Vocology. 2012a. No prelo.

Camargo Z, Tsuji DH, Madureira S. Analysis of dysphonic voices based on the interpretation of acoustic, physiological and perceptual data. In: Pelethorpe S, Tabain M, editors. Proceedings of 6th Internacional Seminar on Speech Production; 2003 Set 7-10. Sidney. Sidney: Macquaire University; 2003. v. 1, p. 31-6.

Camargo Z, Madureira S. Dimensões perceptivas das alterações de qualidade vocal e suas correlações aos planos da acústica e da fisiologia. DELTA - Documentação de Estudos em Linguística Teórica e Aplicada. 2009;25:285-317.

Camargo Z. Fonoaudiologia na interface com as ciências da fala: reflexões acerca dos distúrbios de fala e das possibilidades de cura. In: Marquezan I, organizadora. Motricidade Orofacial: como atuam os especialistas. 1 ed. São José dos Campos: Pulso Editorial; 2004. p. 251-4.

Camargo Z. Análise da qualidade vocal de um grupo de indivíduos disfônicos: uma abordagem interpretativa e integrada de dados de natureza acústica, perceptiva e eletroglotográfica. [Tese de doutorado]. São Paulo: Pontifícia Universidade Católica de São Paulo; 2002.

Camargo Z, Madureira S, Pessoa A, Rusilo LC. Voice quality and gender: some insights on correlation between perceptual and acoustic dimensions In: Ma Q, Ding H, Hirst D, editors. 6th International Conference on Speech Prosody; 2012 Mai 22-25; Shangai. Shangai: Tongji University Press, 2012a. v. 1, p. 115-8.

Camargo Z, Madureira S. The acoustic analysis of speech samples designed for the Voice Profile Analysis Scheme for Brazilian Portuguese (BP-VPAS): long-term f0 and intensity measures. In: 
Botinis A, editor. Proceedings of the Workshop on Experimental Linguistics Athens, Greece; 2010 Ago 25-27. Atenas: ISCA and the University of Athens; 2010. v. 1, p. 33-6.

Camargo Z, Rusilo LC, Madureira S. Evaluating speech samples designed for the Voice Profile Analysis Scheme for Brazilian Portuguese. In: Botinis A, editor. Proceedings of the Fourth ISCA Tutorial and Research Workshop on Experimental Linguistics. 2011 Mai 25-27; Paris. Paris: ISCA; 2011. p. 55-8.

Cukier-Blaj S, Camargo Z, Madureira S. Long-term average spectrum loudness variation in speakers with asthma, paradoxical vocal fold motion and without breathing problems In: Barbosa P, Reis C, Madureira S, editors. Fourth Conference on Speech Prosody; 2008 Mai 6-9; Campinas. Campinas: Capes, Fapesp, CNPq; 2008. v. 1, p. 41-4.

Cukier-Blaj S. Qualidade vocal em indivíduos asmáticos com e sem disfunção paradoxal de pregas vocais: correlatos perceptivo-auditivos, acústicos e fisiológicos. [Dissertação de mestrado]. São Paulo: Pontifícia Universidade Católica de São Paulo; 2006.

Fant G. Acoustic theory of speech production. 2 nd ed. Paris: Mouton; 1970.

Fernandes AC. Descrição da qualidade de voz por meio de proposta de avaliação com motivação fonética. [Dissertação de mestrado]. São Paulo: Pontifícia Universidade Católica de São Paulo; 2011.

Flanagan JL. A difference limen of vocal formant frequency. Journal of the Acoustical Society of America. 1955;27:613-7.

Giannini PP. Distúrbio de voz relacionado ao trabalho docente: um estudo caso-controle. [Tese de doutorado]. Campinas: Universidade Estadual de Campinas; 2010.

Gregio FN, Camargo Z. Dados do tempo de início de sonorização (VOT) na avaliação do sinal vocal de indivíduos com paralisia unilateral de prega vocal. Distúrbios da Comunicação. 2005;17:289-97.

Gregio FN, Queiroz RM, Sacco ABDF, Camargo Z. O uso da eletroglotografia na investigação do vozeamento em adultos sem queixa de fala. Revista Intercâmbio. 2011;23:88-105.

Gregio FN. Configuração do trato vocal supraglótico na produção das vogais do português brasileiro: dados de imagens de ressonância magnética. [Dissertação de mestrado]. São Paulo: Pontifícia Universidade Católica de São Paulo; 2006.

Gregio FN, Gama-Rossi A, Madureira S, Camargo Z. Modelos teóricos de produção e percepção da fala como um sistema dinâmico. Revista CEFAC. 2006;8(2):244-7.

Johnson K. Acoustic \& auditory phonetics. Malden: Blackwel; 2003.

Kreiman J, Gerratt BR. Measuring vocal quality. In: Kent M, Ball B, editors. Voice auality measurement. San Diego: Singular-Thomson Learning; 2000. p. 73-102. 
Laver J. The phonetic description of voice quality. Cambridge: Cambridge University Press; 1980.

Laver J. The phonetic evaluation of voice quality. In: Kent RD, Ball MJ, editors. Voice quality measurement. San Diego: Singular Publishing; 2000. p. 37-48.

Laver J, Mackenzie-Beck J. Vocal Profile Analysis Scheme - VPAS. Speech Science Research Centre. Edinburgh: Queen Margareth University College; 2007.

Laver J, Wirs S, Mackenzie J, Hiller SM. A perceptual protocol for the analysis of vocal profiles. Work in Progress. 1981;14:139-55.

Lima MFB. Sintomas vocais, alteração de qualidade vocal e laríngea em professores. [Dissertação de mestrado]. São Paulo: Pontifícia Universidade Católica de São Paulo; 2008.

Lima-Gregio AM, Barbosa PA. Laryngealizations in cleft and non-cleft speech: acoustics and prosodic considerations. In: Hasegawa-Johnson M, editor. Proceedings of the Speech Prosody; 2010 Mai 11-14; Chicago. 2010. p. 1-4.

Lima-Silva MFB. Avaliação da qualidade vocal com motivação fonética: análise integrada de dados de percepção e acústica. [Tese de doutorado]. São Paulo: Pontifícia Universidade Católica de São Paulo; 2012.

Mackenzie-Beck J. Perceptual analysis of voice quality: the place of vocal profile analysis. In: Hardcastle WJ, Mackenzie-Beck J. A figure of speech: a festschrift for John Laver. Mahwah, NJ: Lawrence Erlbrum Associates; 2005. p. 285-322.

Madureira S. An acoustic study of sequences of words with adjacent primary-stressed syllables: does stress shift occur in Brazilian Portuguese? Cadernos de Estudos Lingüísticos, 2002;43:109-126.

Madureira S. Pitch patterns in Brazilian Portuguese. In: Togneri R, editor. Proceedings of the 5th Australian International Conference on Speech Science and Technology; 1994 Dez 6-8; Perth, Australia. Sydney: Australian Speech Science \& Technology Association; 1994. v. 1, p. 156-8.

Madureira S, Barzaghi L, Mendes B. Voicing contrasts and the deaf: production and perception issues. In: Windsor F, Kelly L, Hewlett N, editors. Themes in clinical phonetics. 1 ed. Mahwah, NJ: Lawrence Erbaum Associates; 2002. p. 417-28.

Madureira S, Diniz J. Qualidade vocálica e fronteiras prosódicas. Intercâmbio. 2002;11:173-9.

Madureira S, Fontes MAS. Deslocamento acentual: um estudo experimental. Intercâmbio. 2003;12:369-75.

Madureira S, Gama-Rossi A, Barbosa PA, Fontes MAS. Brazilian Portuguese and European Portuguese constrasted: an experimental acoustic study of speech segments in clash and non-clash conditions. In: Gussenhoven C, editor. International Conference on Tone and Intonation; 2004 Set 911; Santorini. 2004. p. 10-20. 
Marchesan IQ. Alterações de fala de origem músculo-esquelética. In: Ferreira LP, Befi-Lopes DM, Limongi SCO. Tratado de fonoaudiologia. São Paulo: Roca; 2004. p. 292-303.

Marchesan IQ. Alterações de fala músculo-esqueléticas: possibilidades de cura. In: Marquezan I, organizadora. Comitê de Motricidade Orofacial. Motricidade Orofacial - como atuam os especialistas. São José dos Campos: Pulso Editorial; 2004a. p. 243-9.

Marchesan IQ, Oliveira LR, Svicero MAF, Madureira S, Camargo Z. Análise acústica da produção das vogais pré e pós frenectomia lingual In: Perissinoto J, Mansur L, organizadoras. $19^{\circ}$ Congresso Brasileiro e $8^{\circ}$ Internacional de Fonoaudiologia; 2011 Out 30 - Nov 2; São Paulo. Revista da Sociedade Brasileira de Fonoaudiologia - Suplemento Especial. São Paulo: Sociedade Brasileira de Fonoaudiologia, 2011. v. 16, p. 1409.

Mathieson L. Normal-disordered continuum. In: Kent RD, Ball MJ, editors. Voice quality measurement. San Diego: Singular Publishing; 2000. p. 3-12.

Mendes BCA. Estudo fonético-acústico das vogais do português brasileiro: dados da produção e percepção da fala de um sujeito deficiente auditivo. [Tese de doutorado]. São Paulo: Pontifícia Universidade Católica de São Paulo; 2003.

Oliveira LR. Correlatos acústicos e perceptivos da postura de língua na produção de fala de respiradores orais. [Dissertação de mestrado]. São Paulo: Pontifícia Universidade Católica de São Paulo; 2011.

Pereira LCK. A percepção de plosivas alveolares na produção de um sujeito com deficiência auditiva: um estudo fonético-acústico. [Dissertação de mestrado]. São Paulo: Pontifícia Universidade Católica de São Paulo; 2007.

Pessoa NA, Pereira LK, Madureira S, Camargo ZA. Achados supra-segmentais na fala de um sujeito com deficiência auditiva usuário de implante coclear. In: $2^{\circ}$ Colóquio Brasileiro de Prosódia da Fala; 2009 Jun 4-5. Campinas: UNICAMP; 2009. sem página (CD-ROM).

Pessoa A, Novaes BCAC, Pereira LCK, Camargo Z. Dados de dinâmica e qualidade vocal: correlatos acústicos e perceptivo-auditivos da fala em criança usuária de implante coclear. Journal of Speech Sciences. 2011;1:17-33.

Pessoa A, Novaes BCAC, Madureira S, Camargo Z. Perceptual and acoustic correlates of a speech in a bilateral cochlear implant user. In: 6th International Conference on Speech Prosody; 2012; Shangai. Shangai: Tongji University Press; 2012a. v. 1. p. 51-4.

Rusilo LC, Camargo Z, Madureira S. The validity of some acoustic measures to predict voice quality settings: trends between acoustic and perceptual correlates of voice quality. In: Botinis A, editor. Proceedings of the Fourth ISCA Tutorial and Research Workshop on Experimental Linguistics; 2011 Mai 25-27; Paris. Paris: ISCA; 2011. p. 115-8. 
Silva AHP, Albano E. Brazilian Portuguese rhotics and the phonetics/phonology boundary. In: Ohala JJ, Hasegawa Y, Ohala M, Granville D, Bailey AC, editors. International Congress of Phonetic Sciences - ICPhS; 1999 Ago 1-7; San Francisco. San Francisco: University of California; 1999, p. 3. Soares MFP. Estratégias de produção de fricativas: análise acústica da fala de sujeitos portadores da doença de Parkinson. [Tese de doutorado]. Campinas: Universidade Estadual de Campinas; 2009.

Vieira MN, McInnes FR, Jack MA. On the influence of laryngeal pathologies on acoustic and electroglottographic jitter measures. Journal of the Acoustical Society of America. 2002; 111:1045-55.

Vieira JM, Barbosa PA, Pegoraro-Krook MI. A pausa na produção da fala com comprometimento neurológico. Revista de Estudos da Linguagem. 2004;12(2):181-91.

Whalen DH. Three lines of evidence for direct links between production and perception in speech. In: Ohala JJ, Hasegawa Y, Ohala M, Granville D, Bailey AC, editors. International Congress of Phonetic Sciences - ICPhS; 1999 Ago 1-7; San Francisco. Berkeley: University of California; 1999. vol. 2, p. 1257.

Xu Y. In defense of lab speech. Journal of Phonetics. 2010;38:329-33. 